\title{
"Potential exposure" in practice
}

\author{
J. VALENTIN*
}

ABSTRACT Potential exposures are not normally expected to occur, but they can be anticipated, and the probability of their occurrence can be forecast. Early ICRP thoughts about this concept were developed in considerations about final disposal of long-lived radioactive waste, and about major accidents such as nuclear disasters. ICRP has now examined a third situation: accidents affecting one or a few persons only (but sometimes with severe consequences for those affected). Protection against such potential exposures starts with a structured analysis of scenarios. Given probabilities derived from such analyses, the expected detriment from potential exposures can be computed. This risk can be compared to a risk constraint, which need not be particularly complicated.

\section{The concept of potential exposure}

According to ICRP Publication 60 (ICRP, 1991), normal exposure from a practice is expected to occur with near certainty, from operations conducted as planned or from unintended, high probability but low consequence events. In contrast, potential exposure is not certain to occur. It results from unplanned events such as equipment failure or departure from planned operating procedures. Such events cannot be predicted in detail, but they can be theoretically predicted and a probability of their occurrence can be assigned.

Dose limits do not apply to potential exposures. They must be supplemented by risk constraints. The theoretical basis for such a tool was developed in ICRP Publication 64 (ICRP, 1993). The new ICRP Publication 76, (ICRP, 1997), is aimed at demonstrating how that tool could be applied in practice in "limited accidents" such as unsafe entry into an irradiation room.

The number of individuals affected is small in such cases. Detriment is largely limited to health effects to the persons actually exposed. The processes leading to potential exposure are relatively simple, and may be the predominant threat associated with the practice. 
In contrast, large disasters such as major nuclear accidents invoke detriment beyond health effects to exposed persons (agricultural restrictions, food consumption controls, etc., and vast costs). INSAG (1995), the International Nuclear Safety Advisory Group, has discussed such problems. Final disposal of long-lived waste adds a further dimension of potential exposures in the far future. This was discussed in ICRP Publication 46 (ICRP, 1985).

\section{Criteria of acceptability}

In Publications 60 and 64, ICRP recommended that health risks due to potential exposures be limited and constrained to the same order of magnitude as that implied by dose limits and constraints for normal exposures. These health risks involve two different probability distributions. First, the exposure occurs with a specific probability, say, $P$. Given the exposure, the conditional probability of stochastic and/or deterministic health detriment is a function of the dose, say, $f(\mathrm{E})$. The unconditional probability of health detriment is therefore the product of these two probabilities, i.e. $P \cdot f(\mathrm{E})$. This quantity should be kept at or lower than a reference risk, say, R. For the limited accidents discussed here, $\mathbf{R}$ can be regarded as a source-related individual risk constraint.

The magnitude of the risk constraint will not necessarily be set once and for all. It could be case specific and may be adjusted for various reasons. However, ICRP Publication 76 provides a generic risk constraint which could be used as a starting point when case specific constraints are chosen. This generic constraint is based on an annual effective dose of $5 \mathrm{mSv}$. In many optimised operations, maximum normal annual doses are in that order. Using the cancer death risk coefficient for occupational exposure of $4 \times 10^{-2} \mathrm{~Sv}^{-1}$, the generic reference risk of death $\mathbf{R}$ becomes $2 \times 10^{-4} \mathrm{a}^{-1}$.

It is then possible to assess whether the probability $P$ of unsafe events is acceptably low, and whether the engineered safety and operational procedures of the installation are acceptable. For instance, if the event under study is unsafe entry into a radiation room, where dose rates are high enough that death will always follow an entry (i.e. $f(\mathrm{E})=1$ ), the probability $P$ should be kept less than $\mathbf{R}$. If upon analysis $P$ is found to exceed $\mathbf{R}$, further steps need to be taken to improve safety.

\section{Identification and structuring of scenarios}

An analysis aimed at estimating the probability of occurrence of unsafe events, $P$, needs to identify all types of scenarios that could contribute significantly to potential exposure. A structured approach is advisable. There are several techniques to formalise the analysis, such as Hazard and Operability Studies, and Failure Modes and Effects Analysis. 
In each scenario, demands will be placed on a protection system. Analysis aims at determining whether the system fails when demanded. Demands will be both "normal" actions that routinely challenge the system and "random" events (human or equipment failures). Once all relevant scenarios are believed to be listed, their logical structure must be determined. This type of analysis is well known in engineering. Usually, such structuring is presented in event trees or fault trees.

Event trees start with an initiating demand on a system, and move through successive responses of the system, describing the outcome in terms of success or failure of individual steps and devices. Fault trees begin at the other end, with a specified unwanted outcome, and work backward to analyse possible ways in which this outcome could have occurred.

Conceptually, mathematical analysis of event or fault trees is simple, being based on elementary probability theory. However, the practical application of this theory is often quite difficult because of the many alternative outcomes under study, and in all but the simplest cases computer processing is recommended for correct computation.

\section{Some complications}

If modifications of proven designs are contemplated "in the field", there is considerable risk of potential exposure because of a high probability of human error. Except for pressing circumstances where immediate action is necessary, no modification should be performed until a thorough safety assessment has been carried out and the proposed modification and the assessment are clearly documented.

Sometimes, reduction of a particular type of exposure may be associated with a trade-off in the shape of an increase of other exposure. For instance, inspections are performed regularly in order to detect defects before they cause component failure. An increased inspection frequency of, say, an accelerator may improve the rate of early detection of defects and therefore decrease the probability of potential exposure. However, due to the radiation fields present in such an installation, it would also entail increased occupational exposure. Both types of exposure must therefore be taken into account in an optimisation.

In medical radiation usage, an added complication is that not only excessive doses but also too small doses to the patient may be unsafe. This may preclude comparisons of expected detriment with the risk criterion R. However, structured analysis is still useful in order to identify major contributors of risk. Measures that might be taken to reduce risk to the patient can then be contemplated.

Human errors are often the most significant contributors to potential exposure. Unfortunately, they are also among the most difficult to quantify. They 
are strongly dependent on the situation and so-called performance shaping factors (layout of the workplace, amount of noise and distraction, level of stress, etc.). The probability of repeated human error may also increase with time, if on a first occasion a given error did not lead to untoward consequences.

The above considerations concern the probability $P$ of exposure. The probability $f(\mathrm{E})$ of detriment, given a dose, may also involve complications. For instance, potential exposure situations may entail doses high enough to cause certain death due to deterministic effects. This introduces the possibility of a greater loss of years of life than that associated with stochastically caused death, which occurs later in life. However, no additional weighting for early death seems warranted, in view of the general uncertainty in the probability calculations.

Furthermore, in some practices and operational environments, localised deterministic effects from potential exposures will be the dominating threat. For exposures within present dose limits, deterministic effects are well nigh impossible. Therefore, ICRP has expressly chosen not to take such detriment into account for normal exposures. However, for potential exposure it will be essential to assess detriment from deterministic effects. At the same time, while certainly worth assessing, loss of a finger, say, can obviously not be equated to death. Thus, weighting for severity is required. A generic weighting factor of 0.25 is suggested in ICRP Publication 76, based on various insurance and compensation schemes and considering the need for a reasonably simple generic weighting factor.

\section{REFERENCES}

ICRP (1985) Radiation protection principles for the disposal of solid radioactive waste. ICRP Publication 46. Ann. ICRP, 15, 4.

ICRP (1991) 1990 Recommendations of the International Commission on Radiological Ptotection; ICRP Publication 60. Ann. ICRP, 21, 1-3.

ICRP (1993) Protection from potential exposure: A conceptual framework. ICRP Publication 64, Ann. ICRP, 23, 1.

ICRP (1997) Protection from potential exposures: Application to selected radiation sources, ICRP Publication 76. Ann. ICRP, 27, 2.

INSAG (1995) Potential exposure in nuclear safety. Vienna, IAEA, INSAG-9. 